\title{
Hypotony as a Hazard of Trabeculectomy with Mitomycin C
}

\section{Elizabeth McElnea*, Emer Henry and John Stokes}

Ophthalmology Department, Waterford Regional Hospital, Ardkeen, Co. Waterford, Ireland

*Corresponding Author: Elizabeth McElnea, Specialist Registrar in Ophthalmology,MB BCh BAO, Mater Misericordiae University Hospital, Eccles Street, Dublin 7 , Ireland, Tel: 0035386 8528074; E-mail: mcelneaelizabeth@gmail.com

Received date: October 27, 2015; Accepted date: December 10, 2015; Published date: December 17, 2015

Copyright: (c) 2015 McElnea E, et al. This is an open-access article distributed under the terms of the Creative Commons Attribution License, which permits unrestricted use, distribution, and reproduction in any medium, provided the original author and source are credited.

\section{Introduction}

This report describes the difficulties faced in the management of a case of hypotony following trabeculectomy with mitomycin $\mathrm{C}$ in an attempt to emphasize the importance of this common complication and highlight the options available for its treatment.

\section{Case Report}

An eighty two year old Caucasian lady with primary open angle glaucoma attended eye clinic. She was using guttate latanoprost 50 $\mathrm{g} / \mathrm{ml}$, Brimonidine $2 \mathrm{mg} / \mathrm{ml}$ and combined Dorzolamide $20 \mathrm{~g} / \mathrm{ml}$ and Timolol $5 \mathrm{mg} / \mathrm{ml}$.

This lady was myopic with right eye manifest refraction spherical equivalent of -2.00 and left eye manifest refraction spherical equivalent of -8.00 dioptres. The left eye was amblyopic as a result of this anisometropia. This lady had had bilateral uncomplicated cataract extractions by phacoemulsification with intraocular lens implantation and subsequently bilateral neodymium yttrium aluminium garnet or Nd: YAG laser posterior capsulotomies.

This patient had a medical history of pulmonary tuberculosis and pulmonary fibrosis. She also had aortic valve regurgitation and osteoarthritis. Her regular medications were Digoxin $125 \mu \mathrm{g}$, Bumetanide $1 \mathrm{mg}$ and Lisinopril $1 \mathrm{mg}$ daily. She utilized supplementary oxygen for 16 hours daily.

At her examination the visual acuity from this lady's right and better eye was $6 / 12$ and that from her left eye $6 / 60$ on a Snellen chart. Her intraocular pressures were $20 \mathrm{mmHg}$ bilaterally. Both optic discs were cupped with approximate cup: disc ratios of 0.8. At Humphrey visual field testing progressive visual field losses was noted in this lady's right eye as can be seen from Figure 1. Consequently glaucoma surgery was planned.

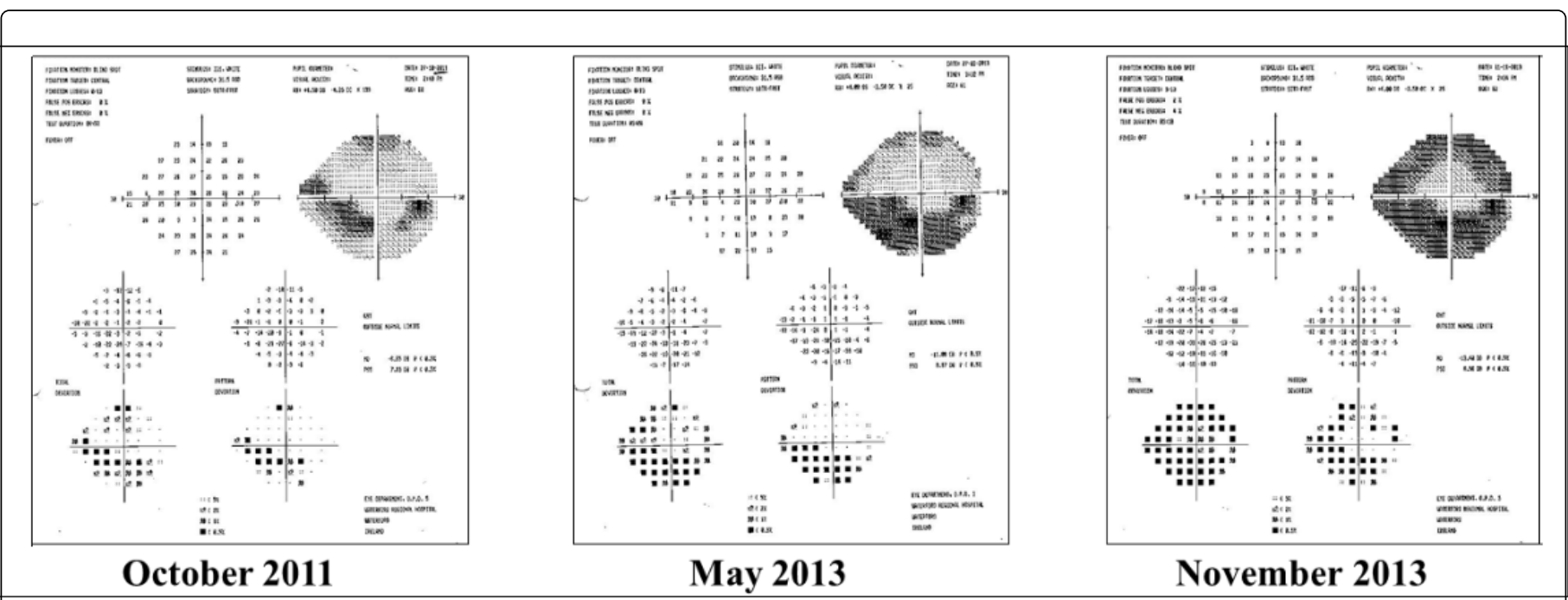

Figure 1: Humphrey Visual fields from our patient's right eye showing glaucomatous visual field losses progressing over a three year period.

This patient subsequently underwent right trabeculectomy wherein three sponges soaked in mitomycin C $0.3 \mathrm{mg} / \mathrm{ml}$ were applied beneath a fornix based conjunctival flap for one minute prior to the creation of a $4 \times 3 \mathrm{~mm}$ limbal based rectangular scleral flap and a $0.4 \times 0.4 \mathrm{~mm}$ sclerotomy. The scleral flap was closed with two 10/0 Nylon sutures. The patient's conjunctiva was noted to be thin and friable and was closed with considerable difficulty at the end of the procedure. This lady was admitted following her surgery, the poor vision from her left, un-operated eye, preventing her safe discharge back to her home.
On the first postoperative day this lady could perceive only hand movements from her right eye. She was noted to now fix with her amblyopic left eye. Her right anterior chamber was shallow. No bleb leak was identifiable. The bleb while shallow superiorly had extended significantly nasally, as can be seen from Figure 2. Choroidal effusions had developed in all four quadrants of the posterior segment. One such effusion can be seen at B-scan ultrasound in Figure 3. 
Page 3 of 6

The addition of the use of the anti-fibrotic drugs 5 -fluoruracil and mitomycin $\mathrm{C}$ to trabeculectomy further improved post-operative intraocular pressure control [3] with the result that adjunctive intraoperative treatment with drugs such as these has become popular not only in eyes at high risk for trabeculectomy failure e.g. eyes with a history of failed glaucoma surgery, aphakic eyes, African-American or Asian eyes and in eyes of patients requiring very low intraocular pressures e.g. those with normal tension glaucoma but also in eyes at relatively low risk for trabeculectomy failure undergoing primary trabeculectomy as in the case of the patient we have described here [1].

Mitomycin C inhibits Tenon's capsule and conjunctival fibroblast proliferation [4]. It was first used in ophthalmology, topically, after pterygium excision, to prevent pterygium recurrence [5]. Its ability to modify the eye's healing response following glaucoma surgery enables a patent filtration fistula to be established. A wide variation in the concentration e.g., $0.02 \mathrm{mg} / \mathrm{ml}-0.5 \mathrm{mg} / \mathrm{ml}$, the duration of exposure e.g., 1-7 minutes and the area to which it is exposed e.g., conjunctiva +/-scleral flap for mitomycin $\mathrm{C}$ has been described for filtration surgery.

\begin{tabular}{|l|l|}
\hline \multirow{3}{*}{ Over-filtration } & Loose closure of the scleral flap \\
\cline { 2 - 3 } & Bleb leak \\
\hline \multirow{2}{*}{ Hypo-secretion of aqueous humour } & Ciliary body shutdown accompanying ocular inflammation \\
\cline { 2 - 3 } & Ciliochoroidal detachment \\
\cline { 2 - 2 } & Formation of cyclodialysis cleft \\
\cline { 2 - 2 } & Mitomycin C ciliary body toxicity \\
\hline
\end{tabular}

Table 1: Causes of hypotony after trabeculectomy.

Ocular hypotension post-trabeculectomy is common and may not affect vision. The term 'hypotony' however is often used to imply that vision is reduced as a result of low intraocular pressure. Prolonged and/or profound hypotony may be responsible for a number of
Histological studies have shown that mitomycin C produces a conjunctival bleb that has a thinner and more irregular epithelium overlying a largely avascular and acellular stroma [6]. Such work also noted breaks in the basement membrane of the basal layer of the conjunctiva and suggested that these areas facilitated aqueous drainage from the surface of the bleb [7]. Indeed the effects of mitomycin C on the conjunctiva following trabeculectomy are clinically evident in the appearance of the bleb created which is more commonly described as thin and avascular [6]. Aqueous humour can frequently be seen to 'ooze' from the conjunctiva of blebs created with mitomycin $\mathrm{C}$ after fluorescein application over the bleb [8].

One complication of glaucoma surgery which has been made all the more frequent by the use of adjunctive antimetabolites is postoperative low intraocular pressure $[9,10]$. Ocular hypotension or hypotony may be defined as an intraocular pressure less than $6 \mathrm{mmHg}$. The hyposecretion of aqueous humour and/or the excessive filtration of aqueous humour is responsible. Either can arise after trabeculectomy for several reasons which have been outlined in Table 1 .

\begin{tabular}{|c|c|}
\hline \multirow{8}{*}{ Complications of Hypotony } & Corneal oedema secondary to corneal de-compensation \\
\hline & Astigmatism \\
\hline & Cataract \\
\hline & Ciliochoroidal effusion producing choroidal detachment \\
\hline & Choroidal haemorrhage \\
\hline & $\begin{array}{l}\text { Hypotonous maculopathy i.e., a low intraocular pressure associated with changes at the fundus } \\
\text { inclusive of cystoid macular oedema, chorioretinal folds, vascular tortuosity and/or optic nerve } \\
\text { head oedema }\end{array}$ \\
\hline & Neuronal atrophy \\
\hline & Phthisis bulbi \\
\hline
\end{tabular}

Table 2: Complications of hypotony.

Occasionally as is depicted in figure 4 a vicious cycle of hypotony, blood-aqueous barrier breakdown, ocular inflammation and ciliary body shut-down which causes a further reduction in aqueous humour structural ocular changes which are shown in Table 2. Many of these are irreversible should they occur and so have the potential to permanently, negatively, impact on vision. production and results in even more profound hypotony can develop [11]. Indeed this is what we hypothesize happened in the case we have described here. 




Table 3: The Treatment of hypotony after trabeculectomy.

Observation may be all that is required in the vast majority of cases of ocular hypotension/hypotony as many are often self-limiting. In one study $70 \%$ of cases of hypotony post trabeculectomy were of less than two weeks duration [12]. The treatment of hypotony becomes more urgent if it is profound, if hypotonous maculopathy which, as already mentioned, may be irreversible, develops, if the anterior chamber flattens resulting in lens-corneal touch which can promote cataract 
progression or if there is poor vision from the fellow eye as in the case we describe here.

Options for the management of hypotony following trabeculectomy are outlined in Table 3. Ideally treatment will eliminate hypotony while preserving filtration function and maintaining, over the long-term, a desirable intraocular pressure [13]. Initial 'conservative' treatment is with topical atropine to prevent pupil block and aqueous suppressants such as topical ß-blockers or oral Acetazolamide which promote healing of the surgically created fistula by temporarily reducing aqueous flow through it [13].

If the above measures prove ineffective temporary tamponade of the conjunctiva by a pressure patch [14] or a large diameter soft bandage contact lens [15] can similarly encourage healing in the area of the fistula. The use of such non-surgical methods is relatively easy and significant complications are rare, but these methods may fail. Additionally, many of the studies describing their use pre-dates the regular use of anti-fibrotic agents and so we are left unsure of their success in treating hypotony following trabeculectomy augmented with agents such as mitomycin $\mathrm{C}$.

In the case we describe here imminent lens-corneal touch necessitated re-formation of the anterior chamber. This can be accomplished with air16 but air often escapes from the anterior chamber into the bleb. Additionally, the placement of air in the anterior chamber can cause endothelial cell loss [17]. We used an ophthalmic viscoelastic device; Healon (Abbott Medical Optics, Illinois United States of America) but as it mixed well with the aqueous fluid it was rapidly cleared from the eye in the excessive aqueous outflow and its effect on the form of the anterior chamber only temporary as a result.

The injection of longer-standing gases such as those used most commonly in retinal surgery for the tamponade of retinal breaks; sulphur hexafluoride $\left(\mathrm{SF}_{6}\right) 18$ and perfluoropropane $\left(\mathrm{C}_{3} \mathrm{~F}_{8}\right)$ [19], for reformation of the anterior chamber following glaucoma surgery has also been described. These gases are not water soluble and expand at high concentrations in an aqueous medium [20] like that of the anterior chamber to form a single bubble that is helpful in both reforming the anterior chamber and sealing the fistula. The main sideeffect of the use of these gases is the development of lens opacities at the area of gas-lens contact [21].

Compression sutures may be placed through the conjunctiva + /-the scleral flap [22]. Compression sutures that are passed through the conjunctiva alone only affect the area of conjunctiva compressed and so may be insufficient in cases such as ours where there is diffuse conjunctival chemosis.

The injection of autologous blood beneath or around an overfiltering bleb may also be an acceptable treatment for hypotony that occurs secondary to excessive filtration after trabeculectomy with adjunctive anti-fibrotic therapy [23]. Blood is useful in that it can affect large areas of conjunctiva. As the goal of injecting blebs with autologous blood is to reduce bleb function the most obvious danger is the creation of a non-functioning bleb [24].

Viscoelastic may be injected into the anterior chamber before the blood to reduce the passage of blood into the anterior chamber and the creation of hyphaema [25]. It is well-known however that occlusion of the trabecular meshwork with a viscoelastic agent may acutely increase intraocular pressure. In glaucomatous eyes, such intraocular pressure spikes can cause a deterioration in visual acuity [26]. Indeed, with or without the prior injection of viscoelastic, patients, particularly those with already advanced visual field losses, need close monitoring after autologous blood injection because viscoelastic in the anterior chamber angle and/or blood flowing under the scleral flap may cause elevations in their intraocular pressure. Intra-bleb autologous platelet injection has also been used effectively for the treatment of bleb overfiltration in at least one patient [27].

Surgical intervention, for which a variety of options exist as can be seen from Table 4, many involving revision of the bleb and/or scleral flap, may eventually be required. The ideal time for such intervention and the ideal surgical technique however remains the subject of ongoing debate.

Confusion exists too regarding the association of postoperative hypotony with later bleb failure. In several series, early i.e. within one month, post-trabeculectomy hypotony was associated with reduced bleb survival time over five years 28,29 . There may be several reasons for this. Tamponade of over-filtering blebs can produce a flat bleb which may promote adhesion formation between the roof and the base of the bleb, thus detrimentally affecting bleb evolution and increasing the risk of later bleb scarring and failure. Hypotony in the early postoperative period can result in an increased breakdown of the blood aqueous barrier as already described. This can in turn, provide for the release of inflammatory mediators into the aqueous and through the filtering bleb into the surrounding tissues. Excessive postoperative inflammation can accelerate wound healing, causing scarring with eventual trabeculectomy failure [30]. When visual acuity has been restored to eyes with hypotonous maculopathy, it has often been achieved by increasing the intraocular pressure to higher than normal levels [31] which defeats the purpose of the initial trabeculectomy and so is of itself undesirable. Finally, bleb-related infection which is associated with eventual filtration failure may occur with greater frequency in dysfunctional blebs.

The management of the case we describe here was challenging. Many cases of hypotony resolve without intervention. In one review of 699 trabeculectomies performed with mitomycin C only nine cases of hypotony in which bleb revision was required were identified. However of those cases of post-surgical hypotony that develop hypotonous maculopathy close to $50 \%$ permanently lose vision [12]. The choice of observation or intervention when the better seeing eye of a patient is affected by hypotony is a difficult one to make. Taking the route of conservative treatment in the case we describe here meant our patient spent a very protracted period of time in hospital. Reducing this patient's topical steroid treatment as suggested, we hoped, would allow for healing of the over-filtering fistula but may well have provided for an increase in intraocular inflammation which could, via its effects on the ciliary body, exacerbate hypotony.

\section{Conclusion}

Prolonged and intractable post-surgical hypotony is rare but the loss of vision which may accompany it is devastating for the patient particularly when he/she may have poor vision from their fellow eye. Further, it may be that even if hypotony resolves over the short term, there persists an increased risk for filtration failure over the longer term. As with much in ophthalmology, prevention is often better than cure and so anti-fibrotic agents should be used as adjuncts in glaucoma surgery judiciously and due attention given to the meticulous closure of both the scleral flap and conjunctiva during trabeculectomy. 


\section{Reasons for Case Selection}

- With the ongoing increase in the life expectancy of patients and an anticipated increase in the size of our aged population we will be increasingly made to consider the appropriateness of trabeculectomy with or without adjunctive anti-metabolites in relatively older patients as in the case described here.

- Hypotony is the most common complication of trabeculectomy with mitomycin C. Its significance is however often overlooked. Herein we hope to emphasize the relatively frequent occurrence of hypotony after trabeculectomy; up to $72 \%$ of trabeculectomies in one series [12], and highlight its possible adverse effects on longerterm surgical outcome.

- While hypotony is common that which requires surgical intervention is uncommon. We wish to describe some of the lesser known' ways in which hypotony can be treated.

- This case indicates that there is much room for improvement in our ability to provide timely, appropriate, convenient follow-up care for our glaucoma patients.

\section{Lessons Learned}

The post-trabeculectomy complication described here may have been prevented by the more judicious use of mitomycin $\mathrm{C}$ in this patient e.g., the application of mitomycin $C$ could have been omitted completely, a lower concentration of the drug could have been utilized, or the duration of exposure of the conjunctiva to this agent reduced.

The social circumstances of the lady we describe in this report necessitated her prolonged in-patient care while she regained her vision postoperatively. In considering the most appropriate care plan for individual glaucoma patients such circumstances should undoubtedly be given more weight.

Patience is a virtue. Management need not always be active. Watchful waiting is often most appropriate.

\section{References}

1. Kurtz S, Leibovitch I (2002) Combined perfluoropropane gas and viscoelastic material injection for anterior chamber reformation following trabeculectomy. Br J Ophthalmol 86: 1225-1227.

2. (1998) The Advanced Glaucoma Intervention Study (AGIS): 4. Comparison of treatment outcomes within race. Seven-year results. Ophthalmology 105: 1146-1164.

3. Palmer SS (1991) Mitomycin as adjunct chemotherapy with trabeculectomy. Ophthalmology 98: 317-321.

4. Jampel HD, Pasquale LR, Dibernardo C (1992) Hypotony maculopathy following trabeculectomy with mitomycin C. Arch Ophthalmol 110: 1049-1050.

5. Jampel HD (1992) Effect of brief exposure to mitomycin C on viability and proliferation of cultured human Tenon's capsule fibroblasts. Ophthalmology 99: 1471-1476.

6. Mietz H, Brunner R, Addicks K, Krieglstein GK (1993) Histopathology of an avascular filtering bleb after trabeculectomy with mitomycin-C. J Glaucoma 2: 266-270

7. Shields MB, Scroggs MW, Sloop CM, Simmons RB (1993) Clinical and histopathologic observations concerning hypotony after trabeculectomy with adjunctive mitomycin C. Am J Ophthalmol 116: 673-683.

8. Belyea DA, Dan JA, Stamper RL, Lieberman MF, Spencer WH (1997) Late onset of sequential multifocal bleb leaks after glaucoma filtration surgery with 5-fluorouracil and mitomycin C. Am J Ophthalmol 124: 40-45.
9. Schubert HD (1996) Postsurgical hypotony: relationship to fistulization, inflammation, chorioretinal lesions, and the vitreous. Surv Ophthalmol 41: 97-125.

10. Costa VP, Wilson RP, Moster MR, Schmidt CM, Gandham S (1993) Hypotony maculopathy following the use of topical mitomycin $\mathrm{C}$ in glaucoma filtration surgery. Ophthalmic Surg 24: 389-394.

11. Watson PG, Jovanovik-Pandova L (2009) Prolonged ocular hypotension: would ciliary tissue transplantation help. Eye (Lond) 23: 1916-1925.

12. Seah SK, Prata JA Jr, Minckler DS, Baerveldt G, Lee PP, et al. (1995) Hypotony following trabeculectomy. J Glaucoma 4: 73-79.

13. Tannenbaum DP, Hoffman D, Greaney MJ, Caprioli J (2004) Outcomes of bleb excision and conjunctival advancement for leaking or hypotonous eyes after glaucoma filtering surgery. Br J Ophthalmol 88: 99-103.

14. Mattox C (1995) Therapeutics and techniques and glaucoma care updates: management of the leaking bleb. J Glaucoma 4: 370-374.

15. Blok MD, Kok JH, van Mil C, Greve EL, Kijlstra A (1990) Use of the Megasoft Bandage Lens for treatment of complications after trabeculectomy. Am J Ophthalmol 110: 264-268.

16. Stewart RH, Kimbrough RL (1980) A method of managing flat anterior chamber following trabeculectomy. Ophthalmic Surg 11: 382-383.

17. Olson RJ (1980) Air and the corneal endothelium: an in vivo specular microscopy study in cats. Arch Ophthalmol 98: 1283-1284.

18. Beigi B, O'Keefe M, Algawi K, Acheson R, Burke J (1997) Sulphur hexafluoride in the treatment of flat anterior chamber following trabeculectomy. Eye (Lond) $11: 672-676$

19. Franks WA, Hitchings RA (1990) Injection of perfluoropropane gas to prevent hypotony in eyes undergoing tube implant surgery. Ophthalmology 97: 899-903.

20. Lincoff A, Haft D, Liggett P, Reifer C (1980) Intravitreal expansion of perfluorocarbon bubbles. Arch Ophthalmol 98: 1646.

21. Franks WA, Hitchings RA (1990) Intraocular gas injection in the treatment of cornea-lens touch and choroidal effusion following fistulizing surgery. Ophthalmic Surg 21: 831-834.

22. Tomlinson CP, Belcher CD 3rd, Smith PD, Simmons RJ (1987) Management of leaking filtration blebs. Ann Ophthalmol 19: 405-408, 411.

23. Wise JB (1993) Treatment of chronic postfiltration hypotony by intrableb injection of autologous blood. Arch Ophthalmol 111: 827-830.

24. Burnstein A, WuDunn D, Ishii Y, Jonescu-Cuypers C, Cantor LB (2001) Autologous blood injection for late-onset filtering bleb leak. Am J Ophthalmol 132: 36-40.

25. Leen MM, Moster MR, Katz LJ, Terebuh AK, Schmidt CM, et al. (1995) Management of overfiltering and leaking blebs with autologous blood injection. Arch Ophthalmol 113: 1050-1055.

26. Savage JA, Thomas JV, Belcher CD 3rd, Simmons RJ (1985) Extracapsular cataract extraction and posterior chamber intraocular lens implantation in glaucomatous eyes. Ophthalmology 92: 1506-1516.

27. Jonas JB, Dugrillon A, Klüter H, Kamppeter B (2003) Subconjunctival injection of autologous platelet concentrate in the treatment of overfiltrating bleb. J Glaucoma 12: 57-58.

28. Benson SE, Mandal K, Bunce CV, Fraser SG (2005) Is post-trabeculectomy hypotony a risk factor for subsequent failure? A case control study. BMC Ophthalmol 5: 7.

29. Stewart WC, Shields MB (1988) Management of anterior chamber depth after trabeculectomy. Am J Ophthalmol 106: 41-44.

30. Skuta GL, Parrish RK 2nd (1987) Wound healing in glaucoma filtering surgery. Surv Ophthalmol 32: 149-170.

31. Cohen SM, Flynn HW Jr, Palmberg PF, Gass JD, Grajewski AL, et al. (1995) Treatment of hypotony maculopathy after trabeculectomy. Ophthalmic Surg Lasers 26: 435-441. 